\title{
Predicting the Local Response of Esophageal Squamous Cell Carcinoma to Neoadjuvant Chemoradiotherapy by Radiomics with a Machine Learning Method Using ${ }^{18}$ F-FDG PET Images
}

\author{
Yuji Murakami ${ }^{1, *}$, Daisuke Kawahara ${ }^{1, *}$, Shigeyuki Tani ${ }^{2}$, Katsumaro Kubo ${ }^{1}$, Tsuyoshi Katsuta ${ }^{1}$, \\ Nobuki Imano ${ }^{1}$, Yuki Takeuchi ${ }^{1}$, Ikuno Nishibuchi ${ }^{1}$, Akito Saito ${ }^{1}$ and Yasushi Nagata ${ }^{1}$ \\ 1 Department of Radiation Oncology, Graduate School of Biomedical \& Health Sciences, Hiroshima University, \\ Hiroshima 734-8551, Japan; kubo1987@hiroshima-u.ac.jp (K.K.); tkatsuta@hiroshima-u.ac.jp (T.K.); \\ imano@hiroshima-u.ac.jp (N.I.); ytake@hiroshima-u.ac.jp (Y.T.); ikuno@hiroshima-u.ac.jp (I.N.); \\ akito@hiroshima-u.ac.jp (A.S.); nagat@hiroshima-u.ac.jp (Y.N.) \\ 2 School of Medicine, Hiroshima University, Hiroshima 734-8551, Japan; b160697@hiroshima-u.ac.jp \\ * Correspondence: yujimura@hiroshima-u.ac.jp (Y.M.); daika99@hiroshima-u.ac.jp (D.K.); \\ Tel.: +81-82-257-1545 (Y.M. \& D.K.); Fax: +81-82-257-1546 (Y.M. \& D.K.)
}

Citation: Murakami, Y.; Kawahara, D.; Tani, S.; Kubo, K.; Katsuta, T.; Imano, N.; Takeuchi, Y.; Nishibuchi, I.; Saito, A.; Nagata, Y. Predicting the Local Response of Esophageal

Squamous Cell Carcinoma to

Neoadjuvant Chemoradiotherapy by Radiomics with a Machine Learning Method Using ${ }^{18}$ F-FDG PET Images. Diagnostics 2021, 11, 1049. https:// doi.org/10.3390/diagnostics11061049

Academic Editor: Luca Boldrini

Received: 9 May 2021

Accepted: 4 June 2021

Published: 7 June 2021

Publisher's Note: MDPI stays neutral with regard to jurisdictional claims in published maps and institutional affiliations.

Copyright: (c) 2021 by the authors. Licensee MDPI, Basel, Switzerland. This article is an open access article distributed under the terms and conditions of the Creative Commons Attribution (CC BY) license (https:// creativecommons.org/licenses/by/ $4.0 /)$.

\begin{abstract}
Background: This study aimed to propose a machine learning model to predict the local response of resectable locally advanced esophageal squamous cell carcinoma (LA-ESCC) treated by neoadjuvant chemoradiotherapy (NCRT) using pretreatment 18-fluorodeoxyglucose positron emission tomography (FDG PET) images. Methods: The local responses of 98 patients were categorized into two groups (complete response and noncomplete response). We performed a radiomics analysis using five segmentations created on FDG PET images, resulting in 4250 features per patient. To construct a machine learning model, we used the least absolute shrinkage and selection operator (LASSO) regression to extract radiomics features optimal for the prediction. Then, a prediction model was constructed by using a neural network classifier. The training model was evaluated with 5-fold cross-validation. Results: By the LASSO analysis of the training data, 22 radiomics features were extracted. In the testing data, the average accuracy, sensitivity, specificity, and area under the receiver operating characteristic curve score of the five prediction models were $89.6 \%, 92.7 \%, 89.5 \%$, and 0.95 , respectively. Conclusions: The proposed machine learning model using radiomics showed promising predictive accuracy of the local response of LA-ESCC treated by NCRT.
\end{abstract}

Keywords: esophageal cancer; squamous cell carcinoma; neoadjuvant chemoradiotherapy; pathological response; machine learning; radiomics

\section{Introduction}

Esophageal cancer is a malignant tumor that still has a poor prognosis. There are two distinct histological types, squamous cell carcinoma and adenocarcinoma, which predominate in East Asia and Western countries, respectively. For patients with resectable locally advanced esophageal squamous cell carcinoma (LA-ESCC), neoadjuvant treatment including neoadjuvant chemotherapy [1] or neoadjuvant chemoradiotherapy (NCRT) [2,3] has been shown to improve survival compared to surgery alone. Therefore, preoperative treatment followed by surgery has been the worldwide standard of care. However, the superiority of neoadjuvant chemotherapy or NCRT has not been determined at this time. On the other hand, definitive chemoradiotherapy (CRT) has been positioned as a treatment option for patients who wish to receive organ-preserving treatment or who are medically inoperable, because the results of definitive CRT to date have been somewhat inferior to those of surgery after neoadjuvant chemotherapy [4]. However, the Japan Clinical Oncology Group (JCOG) 0909 study recently reported that definitive CRT combined with salvage endoscopic resection or salvage surgery showed favorable overall survival and 
esophagectomy-free survival [5]. Thus, organ-preserving treatment will be a promising option for patients with resectable LA-ESCC in the future. However, the choice between surgery-based and organ-preserving definitive CRT-based treatment strategies is still difficult for patients.

In recent years, the introduction of radiomics and artificial intelligence (AI) into medicine has become a major topic of discussion. Radiomics is the study of systematically handling large amounts of imaging information in radiology [6]. The aim of radiomics is generally to extract quantitative, and ideally reproducible, information from diagnostic images, including complex patterns that are difficult to recognize or quantify by the human eye $[7,8]$. By combining radiomics and AI, attempts are being made to perform diagnostic imaging and to predict treatment outcomes [9-13].

18F-fluorodeoxyglucose positron emission tomography (FDG PET) image-derived parameters have been reported to be useful in predicting pathological response to NCRT and prognosis in patients with esophageal cancer [14-16]. In addition, the pathological complete response (pCR) rate after NCRT for resectable LA-ESCC was reported to be comparatively high [2,17]. However, since FDG PET imaging of the therapeutic effect of NCRT is performed immediately before surgery, it has not been possible to select a nonsurgical treatment. We, therefore, devised this study because we thought that if we could predict pathological response with high probability by analyzing medical images before NCRT using radiomics and AI technology, it would contribute to the treatment selection of resectable LA-ESCC patients. The purpose of this study was to propose a model to predict the local pathological response of resectable LA-ESCC patients after NCRT based on FDG PET images before NCRT by radiomics analysis and machine learning.

\section{Materials and Methods}

\subsection{Patients}

Eligibility for this study was based on the following criteria: a histologically confirmed thoracic esophageal or esophagogastric junction cancer; stage IB to IV disease without T4b lesions and distant metastasis other than supraclavicular lymph node metastasis (according to the 7th edition of the Union for International Cancer Control TNM Classification) diagnosed using endoscopy, computed tomography (CT), and FDG PET/CT; and receiving NCRT with cisplatin and 5-fluorouracil (5-FU) followed by surgery from 2003 to 2016. Histopathological response after NCRT was determined according to the 11th edition of the Japanese classification of esophageal cancer [18]. Grade 3 (no viable cancer cells are evident) in this classification corresponds to the pCR in this study. All patients provided written informed consent for treatment. The characteristics of the patients and their tumors are presented in Table 1.

Table 1. Characteristics of patients.

\begin{tabular}{ccc}
\hline Characteristics & \\
\hline Gender & Male/Female & $83 / 15$ \\
Age (year) & Median (range) & $66(35-78)$ \\
Performance status & $0 / 1 / 2$ & $88 / 10 / 0$ \\
Tumor site & Upper/Middle/Lower-EGJ ${ }^{1}$ & $22 / 46 / 30$ \\
T factor & $1 / 2 / 3 / 4$ & $2 / 16 / 79 / 1$ \\
N factor & $0 / 1 / 2 / 3$ & $19 / 52 / 25 / 2$ \\
Clinical stage & IB $/$ II $/$ III/IV & $4 / 21 / 58 / 15$ \\
Local response & $\mathrm{pCR}^{2} /$ non-pCR & $44 / 54$ \\
\hline
\end{tabular}

${ }^{1}$ EGJ = esophagogastric junction, ${ }^{2} \mathrm{pCR}=$ pathological complete response.

\subsection{FDG PET Image Acquisition}

Patients fasted for at least $4 \mathrm{~h}$ before administration of $3.7 \mathrm{MBq} / \mathrm{kg}$ of FDG. The FDG PET/CT images were scanned using a Biograph mCT-64 (Siemens Healthcare, Erlangen, Germany). An unenhanced CT scan was performed with a $3 \mathrm{~mm}$ slice thickness. Both 
$\mathrm{CT}$ and PET scans proceeded under normal tidal breathing. An iterative algorithm with CT-derived attenuation correction was used for the reconstruction of FDG PET images.

\subsection{Neoadjuvant Chemoradiotherapy Followed by Surgery}

Treatment planning CT images were scanned under free breathing using a CT scanner (Light speed RT16; GE Healthcare, Little Chalfont, UK). The slice thickness was $2.5 \mathrm{~mm}$. Three-dimensional radiotherapy treatment planning was performed. We delineated the gross tumor volumes for the primary tumor (GTVp), lymph node metastasis (GTVn), and both (GTVall). For the clinical target volume (CTV), CTVp and CTVn were defined as GTVp and GTVn plus a margin of $5 \mathrm{~mm}$, respectively, and were adjusted according to the anatomical barrier. CTVsub was defined as elective nodal areas for subclinical lymph node metastasis. The elective nodal areas were determined according to primary tumor subsites as follows: supraclavicular to middle mediastinal nodal areas for upper thoracic tumors, upper mediastinal to perigastric nodal areas for middle and lower thoracic tumors, and lower mediastinal to celiac nodal areas for EGJ tumors. The volume including CTVp, CTVn, and CTVsub was defined as CTVall. The planning target volume (PTVall) was defined as the CTVall plus $8-12 \mathrm{~mm}$ margins. We used multiportal beams, if possible, to reduce the dose to the heart. Dose fractionation was $40 \mathrm{~Gy}$ in 20 fractions for PTVall. The concurrent chemotherapy regimen consisted of a combination of cisplatin $\left(70 \mathrm{mg} / \mathrm{m}^{2}\right.$ on days 1 and 29) and 5-FU (700 $\mathrm{mg} / \mathrm{m}^{2} /$ day on days $1-4$ and 29-32). Surgery was performed 4 to 8 weeks after the completion of NCRT. The main surgical procedure was right transthoracic esophagectomy and two-field or three-field lymph node dissection. Patients with upper and middle thoracic esophageal lesions or lymph node metastasis in the upper mediastinum underwent cervical lymphadenectomy.

\subsection{Process of the Radiomics Analysis}

The process for generating a prediction model using a machine learning method with the radiomics feature is shown in Figure 1. The current study was designed as a Transparent Reporting of a Prediction Model for Individual Prognosis or Diagnosis (TRIPOD) type 2a [19].

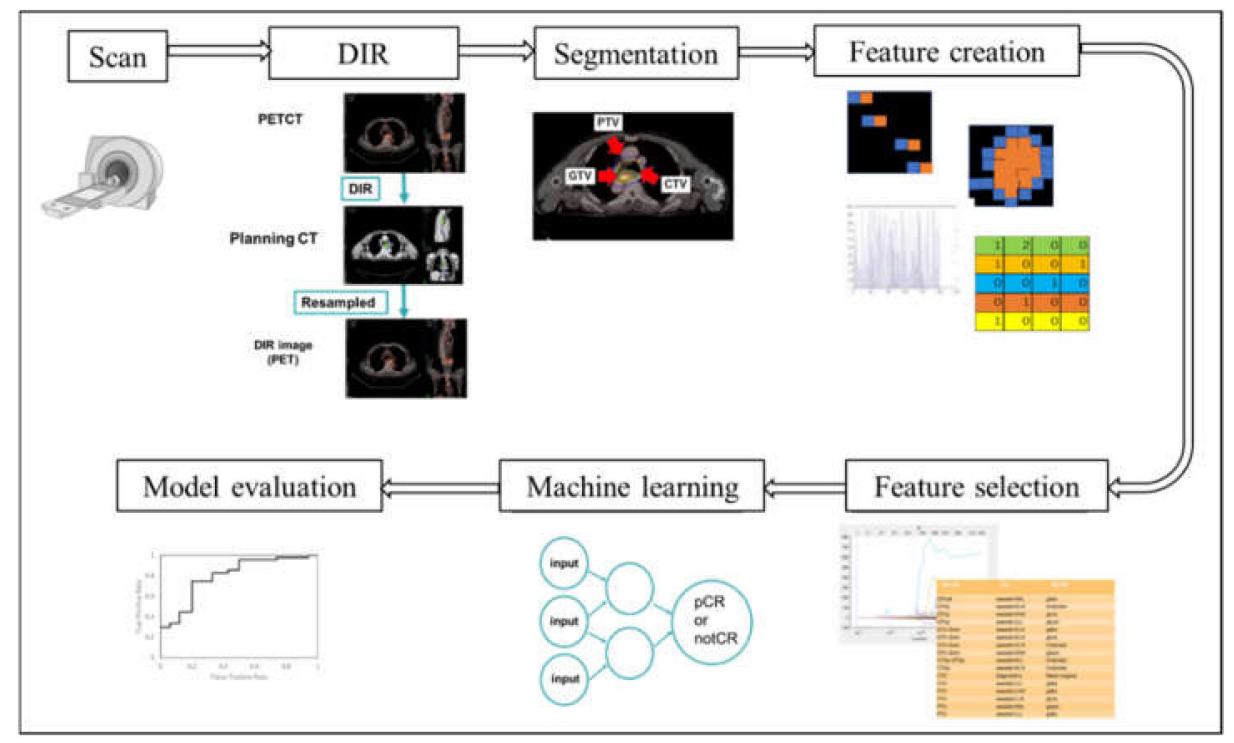

Figure 1. The process of the radiomics analysis and generating prediction model.

\subsubsection{Preparation of Segmentation and Image Registration}

We prepared five segmentations for radiomics analysis: GTVp, GTVp-2 mm, CTVp, CTVp-GTVp, and PTVall. "GTVp-2 mm" was defined as the inner tumor region of GTVp minus $2 \mathrm{~mm}$ of the outer edge. "CTVp-GTVp" was defined as the tumor peripheral region 
of CTVp minus GTVp. For each patient, these segmentations on the treatment planning CT were registered on the FDG PET/CT images using a deformable transformation field. The deformable image registration algorithm for the registration from the treatment planning CT images to the FDG PET/CT images consisted of two steps: a rigid image registration followed by a deformable image registration. By this process, each FDG PET voxel was mapped to a new position based on the transformations used in the CT-CT registration, resulting in a new FDG PET/CT dataset that was deformably registered with the treatment planning CT. All of the segmentations for radiomics analysis were performed by one or two radiation oncologists, including one expert radiation oncologist. Moreover, more than two researchers, including one expert radiation oncologist, checked and confirmed the performance of the deformable image registration and modified the segmentation if necessary.

\subsubsection{Radiomics Analysis}

The pixel values of the FDG PET data were rescaled using the RescaleSlope and RescaleIntercept tags from the DICOM header as follows:

$$
\text { Image Data }=(\text { Image Data }) \times \text { RescaleSlope }+ \text { RescaleIntercept }+1000
$$

The creation of radiomics features was performed using an open-source package in Python, Pyradiomics software [20]. The following features were created: morphologybased features (13 features), first order-based features (18 features), and texture analysis features, including Gray Level Co-occurrence Matrix (GLCM, 24 features), Gray Level Size Zone Matrix (GLSZM, 16 features), Gray Level Run Length Matrix (GLRLM, 16 features), Neighborhood Gray Tone Difference Matrix (NGTDM, 5 features), and Gray Level Dependence Matrix (GLDM, 14 features) (Table 2). Moreover, the FDG PET image data remained unchanged as the original, and these were preprocessed with a wavelet imaging filter. The wavelet filter has low-pass (L) and high-pass (H) filters. The decompositions were constructed in the $\mathrm{x}, \mathrm{y}$, and $\mathrm{z}$ directions. For example, "wavelet-HLL" was interpreted as a wavelet subband image resulting from directional filtering with a high-pass filter along the $\mathrm{x}$-direction $(\mathrm{H})$, a low-pass filter along the $\mathrm{y}$-direction $(\mathrm{L})$, and a low-pass filter along the z-direction (L). In the current study, eight wavelet subband images (wavelet-HLL, wavelet-LHL, wavelet-LHH, wavelet-LLH, wavelet-HLH, wavelet-HHH, wavelet-HHL, and wavelet-LLL) were created (Table 2). Each feature was computed separately with each of the above-mentioned preprocessing steps. From the above, a total of 850 features were created for each segmentation. 
Table 2. Feature type and associated features.

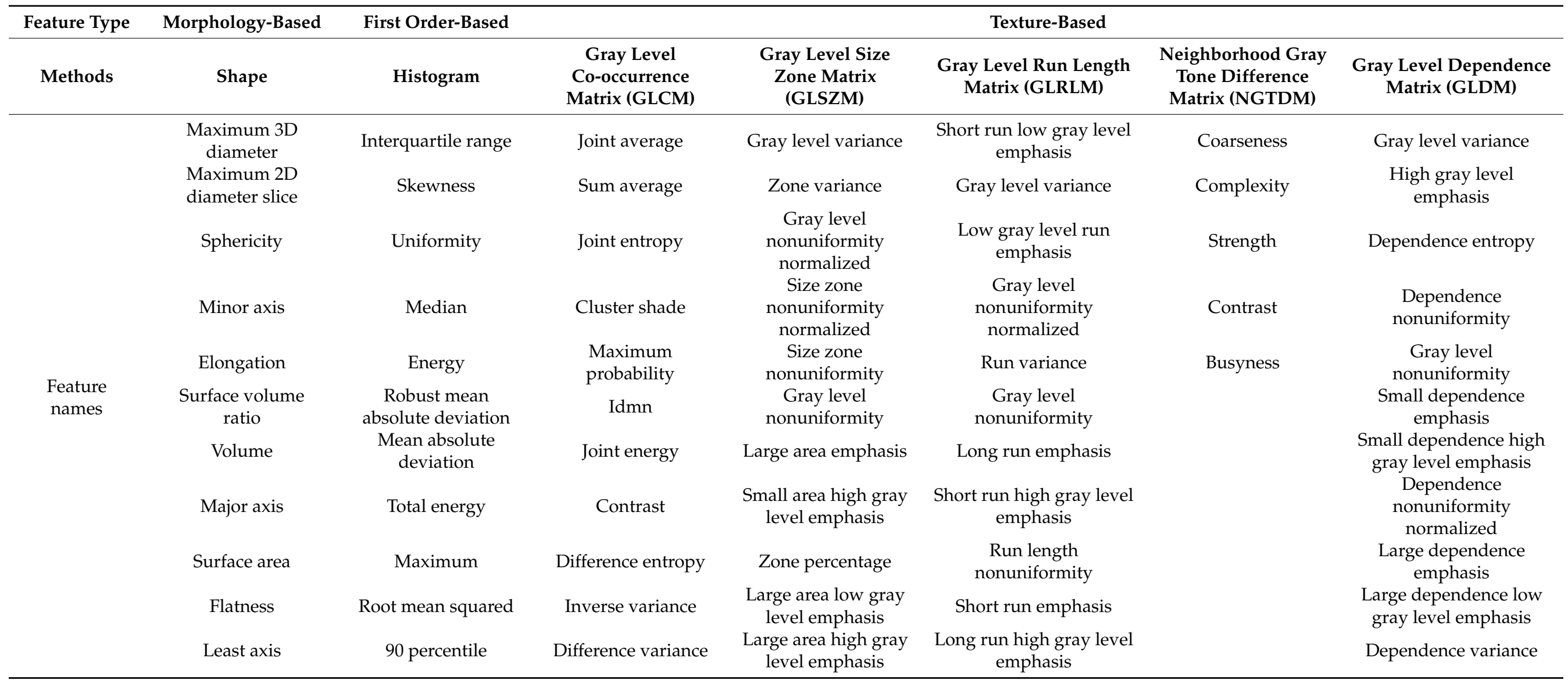


Table 2. Cont.

\begin{tabular}{|c|c|c|c|c|c|c|c|}
\hline Feature Type & Morphology-Based & First Order-Based & \multicolumn{5}{|c|}{ Texture-Based } \\
\hline Methods & Shape & Histogram & $\begin{array}{c}\text { Gray Level } \\
\text { Co-occurrence } \\
\text { Matrix (GLCM) }\end{array}$ & $\begin{array}{l}\text { Gray Level Size } \\
\text { Zone Matrix } \\
\text { (GLSZM) }\end{array}$ & $\begin{array}{l}\text { Gray Level Run Length } \\
\text { Matrix (GLRLM) }\end{array}$ & $\begin{array}{l}\text { Neighborhood Gray } \\
\text { Tone Difference } \\
\text { Matrix (NGTDM) }\end{array}$ & $\begin{array}{l}\text { Gray Level Dependence } \\
\text { Matrix (GLDM) }\end{array}$ \\
\hline & \multirow{12}{*}{$\begin{array}{l}\text { Maximum 2D } \\
\text { diameter column } \\
\text { Maximum 2D } \\
\text { diameter row }\end{array}$} & Minimum & Idn & $\begin{array}{l}\text { High gray level zone } \\
\text { emphasis }\end{array}$ & Run percentage & & $\begin{array}{l}\text { Large dependence high } \\
\text { gray level emphasis }\end{array}$ \\
\hline & & Entropy & $\mathrm{Idm}$ & Small area emphasis & $\begin{array}{c}\text { Long run low gray level } \\
\text { emphasis }\end{array}$ & & $\begin{array}{l}\text { Small dependence low } \\
\text { gray level emphasis }\end{array}$ \\
\hline & & Range & Correlation & $\begin{array}{c}\text { Low gray level zone } \\
\text { emphasis }\end{array}$ & Run entropy & & Low gray level emphasis \\
\hline & & Variance & Autocorrelation & Zone entropy & $\begin{array}{l}\text { High gray level run } \\
\text { emphasis }\end{array}$ & & \\
\hline & & 10 percentile & Sum entropy & $\begin{array}{c}\text { Small area low gray } \\
\text { level emphasis }\end{array}$ & $\begin{array}{c}\text { Run length } \\
\text { nonuniformity } \\
\text { normalized }\end{array}$ & & \\
\hline & & Kurtosis & MCC & & & & \\
\hline & & Mean & Sum squares & & & & \\
\hline & & & Cluster prominence & & & & \\
\hline & & & Imc2 & & & & \\
\hline & & & Difference average & & & & \\
\hline & & & Id & & & & \\
\hline & & & Cluster tendency & & & & \\
\hline Filtering & None & First-orde & istic and texture of & elet decomposition. & omposition levels: LLL, & H, LHL, LHH, HLL, H & H, HHL, HHH. \\
\hline
\end{tabular}




\subsubsection{Construction and Evaluation of Prediction Model}

Among image features created by the radiomics technique, we selected the optimal features for machine learning using the least absolute shrinkage and selection operator (LASSO) logistic regression analysis with MATLAB code [21,22]. Furthermore, we used a machine learning method to construct predictive models, employing a neural network classifier with 10 hidden layers and rectified linear unit activation. The selected radiomics features were used as input values, and the information of "pCR" or "non-pCR" was used as output values. Here, 98 patients were randomly divided into a training group (72 patients; 54 for learning and 18 for validation) and a testing group (26 patients). To find the best predictive models, we used the 5-fold cross-validation method (Figure 2). The training-validation-testing processes were repeated five times for each patient group. The results of the prediction for the training and testing groups were evaluated in terms of accuracy, sensitivity, and specificity. Their predictive performance was evaluated using the area under the receiver operating characteristic (ROC) curve (AUC) score.

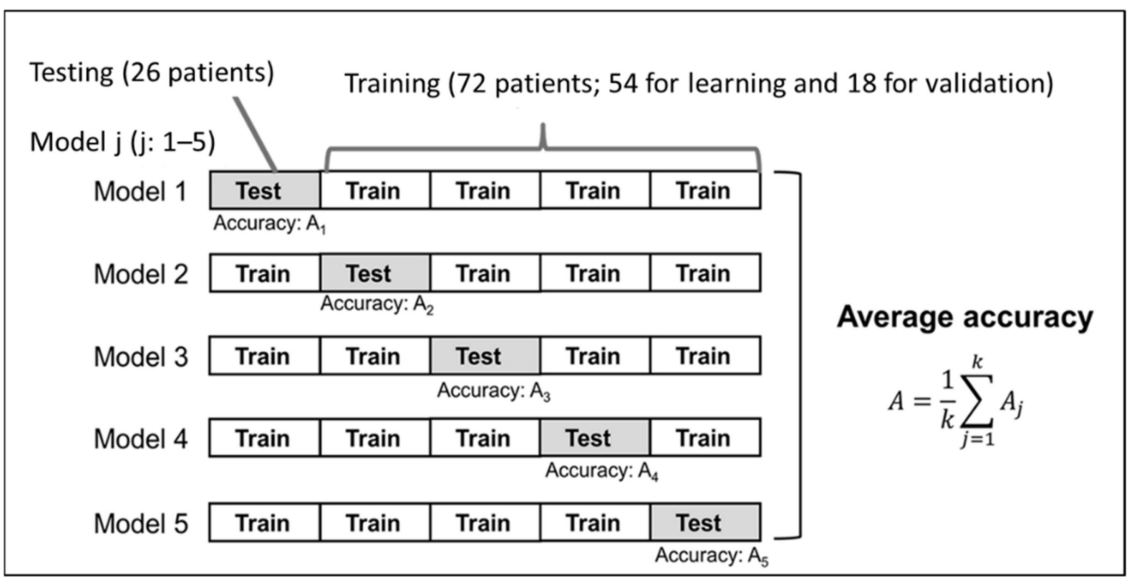

Figure 2. Generation and testing of the prediction model. The proposed neural network model with 5 -fold cross-validation was built in the model training section.

\section{Results}

A total of 4250 features were created from FDG PET images using the radiomics technique. In addition, 22 features for machine learning were selected using the LASSO analysis (Table 3). Twenty-one features were selected from the wavelet filtering features, and one feature was selected from the original image features. Regarding segmentations, eight features were selected from the GTVp, four from GTVp-2 mm, two from CTVp, three from CTVp-GTVp, and five from PTVall. All features were selected from the texture analysis of GLCM, GLRLM, GLSZM, and GLDM. The average values of each feature were compared in the pCR and non-pCR data. The average values of the Gray Level Variance, Gray Level Nonuniformity Normalized, Low Gray Level Run Emphasis, Low Gray Level Zone Emphasis, Gray Level Variance, Small Area Emphasis, Small Area High Gray Level Emphasis, Zone Percentage, Short Run High Gray Level Emphasis, and High Gray Level Run Emphasis in patients with pCR were smaller than those in non-pCR patients. On the other hand, the average values of the Small Area High Gray Level Emphasis, Correlation, MCC, High Gray Level Emphasis, and Large Dependence Low Gray Level Emphasis in pCR patients were higher than those in non-pCR patients. 
Table 3. Selected features by LASSO ${ }^{1}$ regression analysis.

\begin{tabular}{|c|c|c|c|c|}
\hline Segmentation & Filter & Method & Feature Name & Relation of Average Value \\
\hline GTVp $^{2}$ & wavelet-LHH & GLRLM $^{5}$ & Gray Level Variance & $\mathrm{pCR}^{9}<$ non-pCR \\
\hline GTVp & wavelet-LHH & GLRLM & Gray Level Nonuniformity Normalized & pCR < non-pCR \\
\hline GTVp & wavelet-LLH & GLSZM & Gray Level Variance & pCR $<$ non-pCR \\
\hline GTVp & wavelet-LHH & GLRLM & Gray Level Variance & pCR $<$ non-pCR \\
\hline GTVp & wavelet-LHH & GLRLM & Low Gray Level Run Emphasis & pCR > non-pCR \\
\hline GTVp & wavelet-LHH & GLRLM & Gray Level Nonuniformity Normalized & $\mathrm{pCR}>$ non-pCR \\
\hline GTVp & wavelet-LLH & GLSZM $^{6}$ & Gray Level Nonuniformity Normalized & $\mathrm{pCR}>$ non-pCR \\
\hline GTVp & wavelet-HLH & GLSZM & Small Area High Gray Level Emphasis & pCR > non-pCR \\
\hline GTVp-2 mm & original & GLSZM & Low Gray Level Zone Emphasis & pCR $<$ non-pCR \\
\hline GTVp-2 mm & wavelet-HLL & $\mathrm{GLCM}^{7}$ & Correlation & $\mathrm{pCR}>$ non-pCR \\
\hline GTVp-2 mm & wavelet-HLL & GLCM & MCC & pCR > non-pCR \\
\hline GTVp-2 mm & wavelet-HLH & GLSZM & Gray Level Variance & pCR $<$ non-pCR \\
\hline $\mathrm{CTVp}^{3}$ & wavelet-LHL & GLSZM & Gray Level Variance & pCR $<$ non-pCR \\
\hline CTVp & wavelet-LHH & $\mathrm{GLDM}^{8}$ & High Gray Level Emphasis & pCR > non-pCR \\
\hline CTVp-GTVp & wavelet-LLH & GLSZM & Small Area Emphasis & pCR $<$ non-pCR \\
\hline CTVp-GTVp & wavelet-HLH & GLSZM & Small Area High Gray Level Emphasis & pCR $<$ non-pCR \\
\hline CTVp-GTVp & wavelet-HLH & GLSZM & Small Area Emphasis & $\mathrm{pCR}<$ non-pCR \\
\hline PTVall ${ }^{4}$ & wavelet-LHL & GLDM & Large Dependence Low Gray Level Emphasis & pCR > non-pCR \\
\hline PTVall & wavelet-HLH & GLSZM & Zone Percentage & pCR $<$ non-pCR \\
\hline PTVall & wavelet-HHH & GLRLM & Low Gray Level Run Emphasis & pCR > non-pCR \\
\hline PTVall & wavelet-HHH & GLRLM & Short Run High Gray Level Emphasis & $\mathrm{pCR}<$ non-pCR \\
\hline PTVall & wavelet-HHH & GLRLM & High Gray Level Run Emphasis & pCR $<$ non-pCR \\
\hline
\end{tabular}

${ }^{1} \mathrm{LASSO}=$ the least absolute shrinkage and selection operator, ${ }^{2} \mathrm{GTVp}=$ gross tumor volume for primary tumor, ${ }^{3} \mathrm{CTVp}=$ clinical target volume for primary tumor, ${ }^{4}$ PTVall $=$ planning target volume for all targets, ${ }^{5} \mathrm{GLRLM}=$ Gray Level Run Length Matrix, ${ }^{6} \mathrm{GLSZM}=\mathrm{Gray}$ Level Size Zone Matrix,${ }^{7}$ GLCM $=$ Gray Level Co-occurrence Matrix, ${ }^{8}$ GLDM $=$ Gray Level Dependence Matrix, ${ }^{9} \mathrm{pCR}=$ pathological complete response.

Table 4 shows the performance of the NN models. There were five models generated in the 5-fold cross-validation step. The average accuracy of the five models was $95.2 \%$ (range, 92.7-98.2\%) with the training group. The average accuracy, sensitivity, and specificity of the testing group were $89.6 \%$ (range, $87.0-95.7 \%$ ), $92.7 \%$ (range, $84.6-100 \%$ ), and $83.3 \%$ (range, 93.3-100\%), respectively. Figure 3 shows the ROC curves of the predictive performance of five models for the testing group with 5-fold cross-validation. The AUC score was 0.93 for the 1st model, 0.94 for the 2nd model, 0.99 for the 3rd model, 0.92 for the 4th model, and 0.92 for the 5 th model. The average and standard deviation of the AUC score with 5-fold cross-validation were 0.95 and 0.03 , respectively.

Table 4. Model performance (\%).

\begin{tabular}{clcccccc}
\hline \multirow{2}{*}{ Training } & & Model 1 & Model 2 & Model 3 & Model 4 & Model 5 & Average \\
\cline { 3 - 7 } Testing & Accuracy & 94.5 & 92.7 & 96.4 & 94.3 & 98.2 & 95.2 \\
& Accuracy & 87 & 95.7 & 87 & 87 & 91.3 & 89.6 \\
& Sensitivity & 92.3 & 94.1 & 100 & 92.3 & 84.6 & 92.7 \\
& Specificity & 89 & 86 & 100 & 89 & 83.3 & 89.5 \\
\hline
\end{tabular}




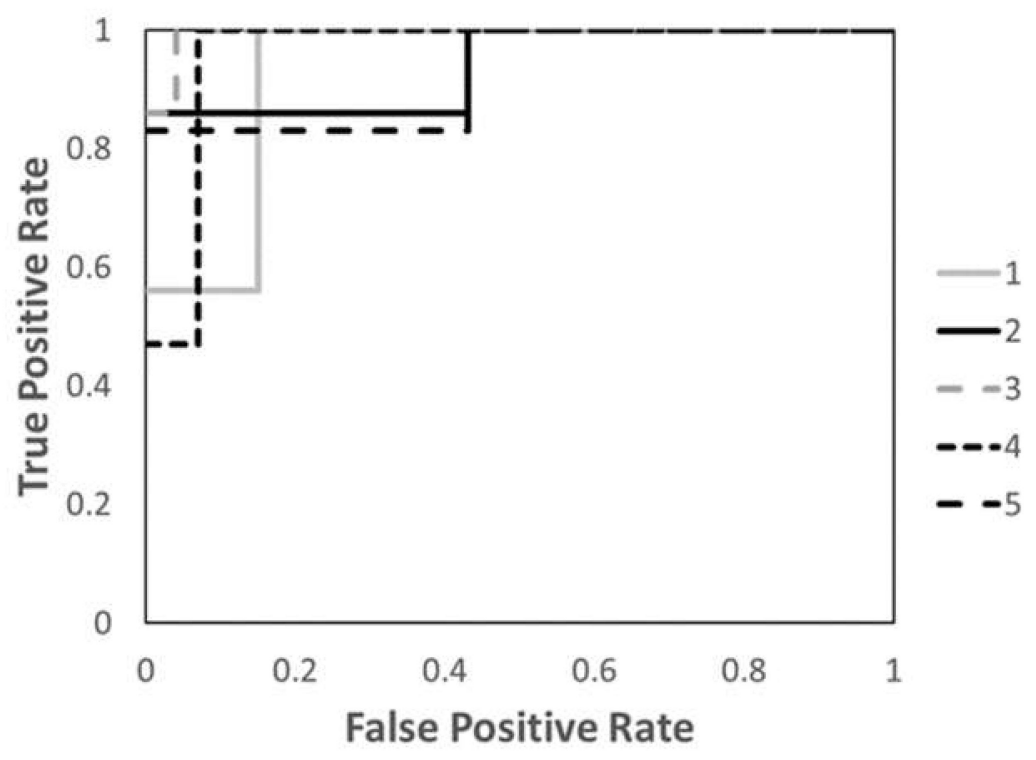

Figure 3. The area under the receiver operator characteristic curve (AUC) of five prediction models constructed by 5 -fold cross-validation method. AUC scores for the models $1-5$ were $0.93,0.94,0.99$, 0.92 , and 0.92 , respectively.

\section{Discussion}

In this study, we constructed a model to predict the pathological response of primary tumors after NCRT for patients with resectable LA-ESCC using FDG PET image-based radiomics and machine learning analysis. Five-fold cross-validation analysis showed promising results with an average prediction accuracy of 89.5\% (87.0-95.7\%) and an average AUC score of 0.95 (0.92-0.99).

For patients with resectable LA-ESCC, neoadjuvant chemotherapy [1] or NCRT [2,3] followed by surgery is the standard treatment. At present, the superiority of NCRT over neoadjuvant chemotherapy remains unclear. To establish the superiority of NCRT over neoadjuvant chemotherapy, two randomized controlled trials were conducted. One was the Japanese three-arm trial, the JCOG1109 NeXT trial [23], for resectable LA-ESCC, and the other was the Irish Neo-AEGIS trial, ICORG10-14 [24], for resectable locally advanced adenocarcinoma. On the other hand, organ-preserving treatment strategies for resectable LA-ESCC have been investigated. Definitive CRT has been positioned as a treatment option for patients who wish to receive organ-preserving treatment or who are medically inoperable because the results of definitive CRT to date have been somewhat inferior to those of surgery after neoadjuvant chemotherapy [4]. However, the JCOG 0909 study recently reported that the 5-year overall survival rate of definitive CRT combined with salvage endoscopic resection or salvage surgery was $64.5 \%$, and the esophagectomy-free survival rate was $54.9 \%$ [5]. Thus, organ-preserving treatment strategy will be a promising option for LA-ESCC patients in the future. However, the choice between surgery-based and organ-preserving, definitive CRT-based treatment strategies is still a very difficult issue for patients.

Regarding the pCR rate after NCRT in resectable LA-ESCC, van Hagen et al. reported that $\mathrm{pCR}$ rates in squamous cell carcinoma and adenocarcinoma were $49 \%$ and $23 \%$, respectively [2]. Our previous study showed that the pCR rate after NCRT for patients with resectable LA-ESCC was $43 \%$ for primary tumors and $35 \%$ for both primary tumors and lymph node metastases [17]. In the patients enrolled in this study, NCRT resulted in pCR of the primary esophageal tumor in 44 of 98 patients (45\%). Patients who achieved pCR after NCRT may have been cured by definitive CRT, and esophageal preservation may have been possible. If we can predict which patients with resectable LA-ESCC can achieve pCR by NCRT based on pretreatment medical information, it may contribute to the treatment selection of patients who wish to preserve their organs. 
In recent years, the introduction of radiomics and AI into medicine has become a major topic. Regarding prediction of treatment outcomes, we reported that the neural network model using the radiomics features of tumor image was more accurate than the visual evaluation method using the image pattern information in predicting the local response of brain metastases to Gamma Knife radiosurgery [9]. Arshad et al., reported that PET image-based radiomics classifiers obtained prior to treatment were useful in predicting the prognosis of patients with non-small-cell lung cancer [10]. Peng et al., reported that PET/CT-based radiomics with deep learning for advanced nasopharyngeal cancer could serve as a prognostic tool and may act as an indicator for individualized induction chemotherapy [11]. Lv et al., reported that radiomics features extracted from the PET and CT components of baseline PET/CT images provide complementary prognostic information and improved outcome prediction for NPC patients compared with the use of clinical parameters alone [12]. Jiang et al., reported that the radiomics signature of PET/CT in gastric cancer patients was a powerful predictor of survivals and could predict which patients could benefit from chemotherapy [13].

FDG PET image-derived parameters have been reported to be useful in predicting the pathological response to NCRT and the prognosis of esophageal cancer patients [14]. We also reported that the rate of decrease in FDG uptake before and after NCRT in FDG PET images could be a prognostic factor $[15,16]$. However, it is necessary to predict the prognosis based on pretreatment information for patients who wish to undergo organpreserving treatment to choose nonsurgical treatment. Regarding the study to evaluate the prediction of treatment response in esophageal cancer by radiomics using medical imaging, studies using CT and FDG PET images have been reported [25-28]. Hou et al. proposed a predictive model of tumor response using the pretreatment contrast-enhanced CT-based radiomics features of 49 patients with esophageal cancer. Their results showed that the classification accuracy using the neural network algorithm for testing cases was 0.917 and the AUC score was 0.800 [26]. Yang et al., developed three CT-based radiomics models for predicting pCR using data of 55 ESCC patients after NCRT. In their study, the AUC score in the testing group was 0.71-0.79 [27]. As for FDG PET imaging, recent studies reported that radiomics features of FDG PET images were better predictors of treatment response than the standard SUV method (SUVmax) [29-31]. Beukinga et al. constructed a model to predict the complete response to NCRT in esophageal cancer based on pretreatment clinical parameters and FDG PET/CT-derived radiomics features and reported that the predictive value of the constructed model was better than that of the SUVmax approach [31]. In their study, when the textual features of radiomics were introduced into the logistic regression analysis, the AUC score was 0.78 compared to 0.58 for the SUVmax model.

The current study constructed a predictive model for pathological findings after NCRT in patients with resectable LA-ESCC with FDG PET image-based radiomics and machine learning. For radiomics analysis, we constructed five segmentations of GTVp, GTVp-2 mm, CTVp, CTVp-GTVp, and PTVall.. Hao et al. developed the tumor shell as a radiomics feature that characterizes the tumor periphery and clarified the correlation between this feature and distant failure [32]. The ingenious things we have done in this study were constructing the inner tumor region (GTVp-2 mm) and tumor peripheral region (CTVpGTVp) as segmentations. Of the 22 features extracted by LASSO analysis, 4 were related to the inner tumor region and 3 were related to the tumor peripheral region. In radiomics analysis, segmentation of not only the target itself but also the tumor peripheral regions and inner tumor regions may contribute to the creation of highly accurate models. Additionally, the LASSO analysis showed characteristic features for pCR and non-pCR cases. The pCR patients had radiomics features of small variance, high homogeneity, and high pixel values in GTVp and inner tumor regions. Moreover, radiomics features of fineness and coarseness by shell analysis can differentiate between $\mathrm{pCR}$ and non-pCR patients. In the CTVp and PTVall regions, the fineness and coarseness of the images were smaller in the pCR patients than in the non-pCR patients. This indicates that the images in and around the tumor of pCR patients were more homogeneous than those of non-pCR patients. Additionally, we 
used a 5-fold cross-validation method to evaluate the prediction model more accurately. As a result, our study showed promising results with a mean prediction accuracy of $89.5 \%$ $(87.0-95.7 \%)$ and a mean AUC score of 0.95 (0.92-0.99). These results were more accurate than the results of previous studies of prediction models for esophageal cancer described earlier. The results also suggest that our prediction method using machine learning of radiomics features of pretreatment FDG PET may be more suitable for predicting $\mathrm{PCR}$.

In radiomics, there are two main categories in imaging features: manually defined features and deep learning features. When compared with manually defined features, deep learning features are more specific to clinical outcomes and data [33]. In the current study, we used manually defined features. Although the results showed promising predictive value, the analysis was based on limited case data from a single institution. In the next step, we will need to validate the prediction model using more case data from multiple institutions. At that time, we plan to use various machine learning methods, including deep learning.

The current study has several limitations. In this prediction model, the pathologic response of only the primary esophageal tumor was considered, and not the pathologic response of lymph node metastases. It is unclear whether similar results will be obtained when the FDG PET imaging system is changed. This study was conducted at a single institution with a limited number of patients. To build a universal prediction model, we consider it necessary to examine a large number of cases in a multicenter setting. Moreover, the robustness of the performance of segmentation and deformable registration was not assessed in the current study. However, this report is significant in that it showed that a predictive model using radiomics and machine learning could significantly change the treatment choice for patients with resectable LA-ESCC in clinical practice in the future.

\section{Conclusions}

We constructed a model to predict the pathological response of primary tumors after NCRT for patients with resectable LA-ESCC using FDG PET image-based radiomics and machine learning, and the model showed promising prediction accuracy.

Author Contributions: Conceptualization, Y.M. and D.K.; methodology, Y.M. and D.K.; validation, Y.N.; formal analysis, Y.M., D.K. and S.T.; investigation, Y.M., D.K. and S.T.; resources, Y.M., D.K. and I.N.; data curation, Y.M., D.K. and S.T.; writing-original draft preparation, Y.M. and D.K.; writing-review and editing, T.K., S.T., K.K., N.I., Y.T., I.N., A.S. and Y.N.; supervision, Y.M. and D.K.; funding acquisition, Y.M. and D.K. All authors have read and agreed to the published version of the manuscript.

Funding: This study was supported by JSPS KAKENHI Grant Number JP19K08153. This study was also supported by Varian Research Grant.

Institutional Review Board Statement: This study was approved by the Institutional Review Board of Hiroshima University Hospital (IRB number: E-1656-1).

Informed Consent Statement: Patient consent to participate was waived due to retrospective study in accordance with the institutional review board.

Data Availability Statement: The data presented in this study are available on request from the corresponding author.

Conflicts of Interest: The authors declare no conflict of interest.

\section{References}

1. Ando, N.; Kato, H.; Igaki, H.; Shinoda, M.; Ozawa, S.; Shimizu, H.; Nakamura, T.; Yabusaki, H.; Aoyama, N.; Kurita, A.; et al. A randomized trial comparing postoperative adjuvant chemotherapy with cisplatin and 5-fluorouracil versus preoperative chemotherapy for localized advanced squamous cell carcinoma of the thoracic esophagus (JCOG9907). Ann. Surg. Oncol. 2011, 19, 68-74. [CrossRef]

2. Van Hagen, P.; Hulshof, M.; Van Lanschot, J.; Steyerberg, E.; Henegouwen, M.V.B.; Wijnhoven, B.; Richel, D.; Nieuwenhuijzen, G.A.; Hospers, G.A.P.; Bonenkamp, J.; et al. Preoperative chemoradiotherapy for esophageal or junctional cancer. N. Engl. J. Med. 2012, 366, 2074-2084. [CrossRef] [PubMed] 
3. Shapiro, J.; Van Lanschot, J.J.B.; Hulshof, M.C.; Van Hagen, P.; Van Berge Henegouwen, M.I.; Wijnhoven, B.P.L.; Van Laarhoven, H.W.M.; Nieuwenhuijzen, G.A.P.; Hospers, G.A.P.; Bonenkamp, J.J.; et al. Neoadjuvant chemoradiotherapy plus surgery versus surgery alone for oesophageal or junctional cancer (CROSS): Long-term results of a randomised controlled trial. Lancet Oncol. 2015, 16, 1090-1098. [CrossRef]

4. Kato, K.; Muro, K.; Minashi, K.; Ohtsu, A.; Ishikura, S.; Boku, N.; Takiuchi, H.; Komatsu, Y.; Miyata, Y.; Fukuda, H. Phase II study of chemoradiotherapy with 5-fluorouracil and cisplatin for stage II-III esophageal squamous cell carcinoma: JCOG trial (JCOG 9906). Int. J. Radiat. Oncol. 2011, 81, 684-690. [CrossRef]

5. Ito, Y.; Takeuchi, H.; Ogawa, G.; Kato, K.; Onozawa, M.; Minashi, K.; Yano, T.; Nakamura, K.; Tsushima, T.; Hara, H.; et al. Final analysis of single-arm confirmatory study of definitive chemoradiotherapy including salvage treatment in patients with clinical stage II/III esophageal carcinoma: JCOG0909. J. Clin. Oncol. 2020, 38, 4545. [CrossRef]

6. Lambin, P.; Rios-Velazquez, E.; Leijenaar, R.; Carvalho, S.; Van Stiphout, R.G.; Granton, P.; Zegers, C.M.; Gillies, R.; Boellard, R.; Dekker, A.; et al. Radiomics: Extracting more information from medical images using advanced feature analysis. Eur. J. Cancer 2012, 48, 441-446. [CrossRef] [PubMed]

7. Gillies, R.J.; Kinahan, P.E.; Hricak, H. Radiomics: Images are more than pictures, they are data. Radiology 2016, $278,563-577$. [CrossRef]

8. Yip, S.S.F.; Aerts, H.J.W.L. Applications and limitations of radiomics. Phys. Med. Biol. 2016, 61, R150-R166. [CrossRef]

9. Kawahara, D.; Tang, X.; Lee, C.K.; Nagata, Y.; Watanabe, Y. Predicting the local response of metastatic brain tumor to gamma knife radiosurgery by radiomics with a machine learning method. Front. Oncol. 2021, 10, 1-8. [CrossRef]

10. Arshad, M.A.; Thornton, A.; Lu, H.; Tam, H.; Wallitt, K.; Rodgers, N.; Scarsbrook, A.; McDermott, G.; Cook, G.; Landau, D.; et al. Discovery of pre-therapy 2-deoxy-2-18F-fluoro-D-glucose positron emission tomography-based radiomics classifiers of survival outcome in non-small-cell lung cancer patients. Eur. J. Nucl. Med. Mol. Imaging 2019, 46, 455-466. [CrossRef]

11. Peng, H.; Dong, D.; Fang, M.-J.; Li, L.; Tang, L.-L.; Chen, L.; Li, W.-F.; Mao, Y.-P.; Fan, W.; Liu, L.-Z.; et al. Prognostic Value of Deep Learning PET/CT-Based Radiomics: Potential Role for Future Individual Induction Chemotherapy in Advanced Nasopharyngeal Carcinoma. Clin. Cancer Res. 2019, 25, 4271-4279. [CrossRef] [PubMed]

12. Lv, W.; Yuan, Q.; Wang, Q.; Ma, J.; Feng, Q.; Chen, W.; Rahmim, A.; Lu, L. Radiomics analysis of PET and CT components of PET/CT imaging integrated with clinical parameters: Application to prognosis for nasopharyngeal carcinoma. Mol. Imaging Biol. 2019, 21, 954-964. [CrossRef] [PubMed]

13. Jiang, Y.; Yuan, Q.; Lv, W.; Xi, S.; Huang, W.; Sun, Z.; Chen, H.; Zhao, L.; Liu, W.; Hu, Y.; et al. Radiomic signature of 18F fluorodeoxyglucose PET/CT for prediction of gastric cancer survival and chemotherapeutic benefits. Theranostics 2018, 8 , 5915-5928. [CrossRef] [PubMed]

14. Westerterp, M.; Van Westreenen, H.L.; Reitsma, J.B.; Hoekstra, O.S.; Stoker, J.; Fockens, P.; Jager, P.L.; Van Eck-Smit, B.L.F.; Plukker, J.T.M.; Van Lanschot, J.J.B.; et al. Esophageal cancer: CT, endoscopic US, and FDG PET for assessment of response to neoadjuvant therapy—Systematic review. Radiology 2005, 236, 841-851. [CrossRef]

15. Hamai, Y.; Hihara, J.; Emi, M.; Furukawa, T.; Yamakita, I.; Kurokawa, T.; Okada, M. Ability of fluorine-18 fluorodeoxyglucose positron emission tomography to predict outcomes of neoadjuvant chemoradiotherapy followed by surgical treatment for esophageal squamous cell carcinoma. Ann. Thorac. Surg. 2016, 102, 1132-1139. [CrossRef]

16. Hamai, Y.; Emi, M.; Ibuki, Y.; Murakami, Y.; Nishibuchi, I.; Nagata, Y.; Furukawa, T.; Kurokawa, T.; Ohsawa, M.; Yoshikawa, T.; et al. Predictions of pathological features and recurrence based on FDG-PET findings of esophageal squamous cell carcinoma after trimodal therapy. Ann. Surg. Oncol. 2020, 27, 1-9. [CrossRef]

17. Murakami, Y.; Hamai, Y.; Emi, M.; Hihara, J.; Imano, N.; Takeuchi, Y.; Takahashi, I.; Nishibuchi, I.; Kimura, T.; Okada, M.; et al. Long-term results of neoadjuvant chemoradiotherapy using cisplatin and 5-fluorouracil followed by esophagectomy for resectable, locally advanced esophageal squamous cell carcinoma. J. Radiat. Res. 2018, 59, 616-624. [CrossRef]

18. Society, J.E. Japanese Classification of Esophageal Cancer, 11th Edition: Part I. Esophagus 2017, 14, 1-36. [CrossRef] [PubMed]

19. Collins, G.S.; Reitsma, J.B.; Altman, D.G.; Moons, K.G.M. Transparent reporting of a multivariable prediction model for individual prognosis or diagnosis (TRIPOD): The TRIPOD statement. Ann. Intern. Med. 2015, 162, 55-63. [CrossRef]

20. Van Griethuysen, J.J.; Fedorov, A.; Parmar, C.; Hosny, A.; Aucoin, N.; Narayan, V.; Beets-Tan, R.G.; Fillion-Robin, J.-C.; Pieper, S.; Aerts, H.J. Computational radiomics system to decode the radiographic phenotype. Cancer Res. 2017, 77, e104-e107. [CrossRef]

21. Tibshirani, R. The lasso method for variable selection in the cox model. Stat. Med. 1997, 16, 385-395. [CrossRef]

22. Zhang, J.-X.; Song, W.; Chen, Z.-H.; Wei, J.-H.; Liao, Y.-J.; Lei, J.; Hu, M.; Chen, G.-Z.; Liao, B.; Lu, J.; et al. Prognostic and predictive value of a microRNA signature in stage II colon cancer: A microRNA expression analysis. Lancet Oncol. 2013, 14, 1295-1306. [CrossRef]

23. Nakamura, K.; Kato, K.; Igaki, H.; Ito, Y.; Mizusawa, J.; Ando, N.; Udagawa, H.; Tsubosa, Y.; Daiko, H.; Hironaka, S.; et al. Three-arm phase III trial comparing cisplatin plus 5-FU (CF) versus docetaxel, cisplatin plus 5-FU (DCF) versus radiotherapy with CF (CF-RT) as preoperative therapy for locally advanced esophageal cancer (JCOG1109, NExT Study). Jpn. J. Clin. Oncol. 2013, 43, 752-755. [CrossRef] [PubMed]

24. Reynolds, J.; Preston, S.; O’Neill, B.; Baeksgaard, L.; Griffin, S.; Mariette, C.; Cuffe, S.; Cunningham, M.; Crosby, T.; Parker, I.; et al. ICORG 10-14: NEOadjuvant trial in Adenocarcinoma of the oEsophagus and oesophagoGastric junction International Study (Neo-AEGIS). BMC Cancer 2017, 17, 1-10. [CrossRef] 
25. Sah, B.-R.; Owczarczyk, K.; Siddique, M.; Cook, G.; Goh, V. Radiomics in esophageal and gastric cancer. Abdom. Radiol. 2019, 44, 2048-2058. [CrossRef] [PubMed]

26. Hou, Z.; Ren, W.; Li, S.; Liu, J.; Sun, Y.; Yan, J.; Wan, S. Radiomic analysis in contrast-enhanced CT: Predict treatment response to chemoradiotherapy in esophageal carcinoma. Oncotarget 2017, 8, 104444-104454. [CrossRef]

27. Yang, Z.; He, B.; Zhuang, X.; Gao, X.; Wang, D.; Li, M.; Lin, Z.; Luo, R. CT-based radiomic signatures for prediction of pathologic complete response in esophageal squamous cell carcinoma after neoadjuvant chemoradiotherapy. J. Radiat. Res. 2019, 60, 538-545. [CrossRef]

28. Paul, D.; Su, R.; Romain, M.; Sébastien, V.; Pierre, V.; Isabelle, G. Feature selection for outcome prediction in oesophageal cancer using genetic algorithm and random forest classifier. Comput. Med. Imaging Graph. 2017, 60, 42-49. [CrossRef]

29. Tixier, F.; Le Rest, C.C.; Hatt, M.; Albarghach, N.; Pradier, O.; Metges, J.-P.; Corcos, L.; Visvikis, D. Intratumor heterogeneity characterized by textural features on baseline 18F-FDG PET images predicts response to concomitant radiochemotherapy in esophageal cancer. J. Nucl. Med. 2011, 52, 369-378. [CrossRef]

30. Nakajo, M.; Jinguji, M.; Nakabeppu, Y.; Nakajo, M.; Higashi, R.; Fukukura, Y.; Sasaki, K.; Uchikado, Y.; Natsugoe, S.; Yoshiura, T. Texture analysis of 18F-FDG PET/CT to predict tumour response and prognosis of patients with esophageal cancer treated by chemoradiotherapy. Eur. J. Nucl. Med. Mol. Imaging 2017, 44, 206-214. [CrossRef] [PubMed]

31. Beukinga, R.J.; Hulshoff, J.B.; Van Dijk, L.V.; Muijs, C.T.; Burgerhof, J.G.; Kats-Ugurlu, G.; Slart, R.H.; Slump, C.H.; Mul, V.E.; Plukker, J.T. Predicting response to neoadjuvant chemoradiotherapy in esophageal cancer with textural features derived from pretreatment 18F-FDG PET/CT imaging. J. Nucl. Med. 2017, 58, 723-729. [CrossRef] [PubMed]

32. Hao, H.; Zhou, Z.; Li, S.; Maquilan, G.; Folkert, M.R.; Iyengar, P.; Westover, K.D.; Albuquerque, K.; Liu, F.; Choy, H.; et al. Shell feature: A new radiomics descriptor for predicting distant failure after radiotherapy in non-small cell lung cancer and cervix cancer. Phys. Med. Biol. 2018, 63, 095007. [CrossRef] [PubMed]

33. Liu, Z.; Wang, S.; Dong, D.; Wei, J.; Fang, C.; Zhou, X.; Sun, K.; Li, L.; Li, B.; Wang, M.; et al. The applications of radiomics in precision diagnosis and treatment of oncology: Opportunities and challenges. Theranostics 2019, 9, 1303-1322. [CrossRef] [PubMed] 